\title{
STOMACH
}

\section{SPECT imaging of the stomach: comparison with barostat, and effects of sex, age, body mass index, and fundoplication}

\author{
E P Bouras, S Delgado-Aros, M Camilleri, E J Castillo, D D Burton, G M Thomforde, \\ H J Chial
}

See end of article for authors' affiliations

.....................

Correspondence to: Dr M Camilleri, Mayo Clinic, Charlton 7-154, 200 First St SW, Rochester, MN 55905, USA;

camilleri.michael@mayo.edu

Accepted for publication 25 June 2002

\begin{abstract}
Background: Impaired gastric accommodation may lead to dyspeptic symptoms. A non-invasive method using single photon emission computed tomography (SPECT) has been developed to measure gastric volumes.

Aims and methods: Our aims were: to assess the accuracy of SPECT with three dimensional image analysis to measure balloon volumes in vitro; to compare gastric barostat balloon volumes measured post-meal and post-distension with total gastric volumes measured simultaneously with SPECT; to present normal gastric volume data for healthy adults; and to compare SPECT data in health with symptomatic post-fundoplication patients.

Results: In vitro balloon volumes measured by SPECT were highly accurate $\left(R^{2}=0.99\right)$. When measured simultaneously by gastric barostat and SPECT, postprandial/fasting volume ratios $(2.2$ (0.12) (mean $(S E M)) \vee 2.3(0.15)$, respectively; $p=0.6)$ and post-distension volume ratios (1.4 (0.1) v1.3 (0.1); $p=0.2$ ) were highly comparable. In females, postprandial gastric volumes (675 (14) v 744 (20) $\mathrm{ml}$ for males; $\mathrm{p}=0.004$ ) and changes in gastric volumes (464 (14) $\mathrm{ml} \mathrm{v} 521$ (20) ml for males; $p=0.01$ ) measured by SPECT were significantly lower than in males. No effects of age or body mass index were noted. The postprandial/fasting gastric volume ratio by SPECT was lower in post-fundoplication patients $(2.7(0.2))$ than in healthy controls $(3.4(0.1) ; p=0.003)$.

Conclusions: SPECT provides a non-invasive estimate of the effect of a meal on total gastric volume that is comparable to changes in balloon volume observed with the gastric barostat. The SPECT technique is promising for investigation of gastric volumes in health and disease and the effects of pharmacological agents.
\end{abstract}

T he postprandial increase in gastric volume results from gastric wall relaxation and is known as gastric accommodation. Postprandial symptoms including early satiety, bloating, and nausea are associated with impaired gastric accommodation in disease states such as functional dyspepsia, rumination syndrome, post-vagotomy/gastric surgery, and diabetes mellitus associated with vagal neuropathy. ${ }^{1-7} \mathrm{~A}$ barostatically controlled balloon placed in the proximal stomach ("gastric barostat") has been regarded as the gold standard for assessment of gastric accommodation. In this method, the intraballoon pressure is clamped, and the change in volume of the infinitely compliant balloon ${ }^{1}$ reflects a change in tone-that is, relaxation.

Although the gastric barostat technique has been used predominantly in small scale research projects, its invasive nature makes it impractical for use in large scale pharmacodynamic studies. In addition, the gastric barostat procedure is not tolerated by many patients, and this limits its usefulness in clinical practice. These considerations led our group to develop a less invasive approach to measure postprandial changes in gastric volume.

Previous studies have shown that technecium-99m $\left({ }^{99 \mathrm{~m}} \mathrm{Tc}\right)$ pertechnetate imaging of the gastric mucosa, as performed in a Meckel's scan, is feasible and can be used to measure gastric wall motion with dynamic scintigraphy. ${ }^{8-10}$ We have combined imaging of the gastric wall (rather than radiolabelling of the meal) using intravenously administered ${ }^{99 \mathrm{~m}} \mathrm{Tc}$ pertechnetate with non-invasive single photon emission computed tomography (SPECT) and three dimensional image analysis to measure total gastric volume. ${ }^{11}$ With this technique, it is possible to measure the volume of the entire stomach and to assess the gastric response to a meal. The SPECT method appears promising as a non-invasive tool for the evaluation of gastric volume changes in response to pharmacological perturbations and in health and disease states. ${ }^{12-16}$ Prior to this study, the results of SPECT imaging of the stomach had not been compared with those of the gold standard barostat method.

The objectives of this study were: (1) to assess the accuracy of SPECT with three dimensional image analysis (SPECTANALYZE) to measure known balloon volumes in vitro; (2) to compare changes in gastric barostat balloon volumes measured post-meal and post-distension with changes in total gastric volumes measured simultaneously with SPECT; (3) to present normal data for fasting and postprandial gastric volumes measured by SPECT in healthy adults, and assess the influences of sex, age, and body mass index on these parameters; and (4) to compare gastric volumes measured in healthy adults with measurements from patients with upper gastrointestinal symptoms post-fundoplication.

\section{METHODS}

\section{Study participants}

All protocols included in this paper were approved by the Mayo Institutional Review Board.

Abbreviations: SPECT, single photon emission computed tomography; ${ }^{99 \mathrm{~m}} \mathrm{Tc}$, technecium- $99 \mathrm{~m}$. 

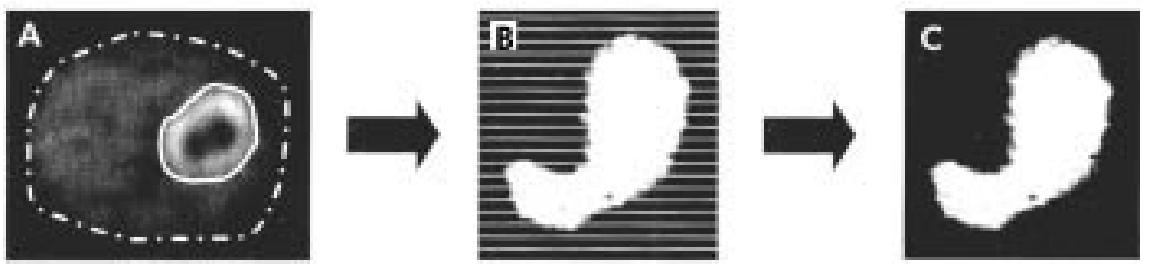

Figure 1 Single photon emission computed tomography (SPECT) data handling and analysis. Multiple transaxial SPECT images (A) were summed and reconstructed via a semiautomated algorithm using the ANALYZE PC 2.5 software system (B) into a three dimensional rendering of the stomach $(\mathrm{C})$ for subsequent measurements of gastric volumes.

\section{Simultaneous SPECT and barostat assessment}

Healthy volunteers aged 18-65 years without current gastrointestinal symptoms or previous gastrointestinal illness were recruited by public advertisement. Exclusion criteria included: prior abdominal surgery other than appendectomy, tubal ligation, or caesarean section; positive symptoms on an abridged bowel symptom questionnaire; use of medications that may alter gastrointestinal function; and use of over the counter medications within seven days of the study.

Eighteen healthy adults were enrolled in the study. One participant was unable to complete the study because of an inability to tolerate the intragastric balloon. A second participant was excluded from analysis due to malfunction of the gastric barostat (kinking of the tube with failed inflation of the intragastric balloon). Data for 16 volunteers (seven females, nine males; mean age 30 (2) years (range 22-48)) were available for analysis.

\section{Normative fasting and postprandial gastric volume data using SPECT in healthy adults}

Normative data for healthy adults were obtained by pooling SPECT data from healthy volunteers who had received placebo medications or served as healthy controls and received no treatment in research protocols previously conducted in our laboratory. ${ }^{12-16}$ All data were analysed by the same technologist (DDB). Healthy volunteers were aged 18-65 years and had been recruited by public advertisement. None of the healthy participants had current gastrointestinal symptoms or previous gastrointestinal illnesses. Exclusion criteria are as listed above.

Data from 73 healthy adults ( 48 females, 25 males; mean age 34.4 (1.1) years (range 18-57)) were included in the analysis. Average height, weight, and body mass index were $1.71(0.01) \mathrm{m}, 86.7(3.2) \mathrm{kg}$, and $29(1) \mathrm{kg} / \mathrm{m}^{2}$, respectively.

\section{SPECT results from patients with upper abdominal symptoms post-fundoplication}

A computerised diagnostic index was used to identify adults with upper abdominal symptoms post-fundoplication who were evaluated in the motility clinic at the Mayo Clinic Rochester and had undergone measurement of gastric volume using SPECT. The study comparing these results with those of healthy controls was approved by the Mayo Institutional Review Board, and authorisation for the use of the medical records for research purposes was confirmed for each individual before accessing the records.

We identified 15 patients (eight females, seven males; mean age 47.9 (3.0) years (range 31-74)) with upper gastrointestinal symptoms post-fundoplication who underwent clinical evaluation and SPECT assessment of gastric volumes.

\section{SPECT-ANALYZE technique to measure gastric volumes}

All tomographic images were acquired using the same large field of view dual headed gamma camera system (SMV SPECT System) equipped with low energy high resolution collimators. Subjects were positioned supine on the imaging table with detectors over the upper and mid abdomen to insure imaging of the stomach. Imaging was started 10 minutes after intravenous injection of $10 \mathrm{mCi}{ }^{99 \mathrm{~m}} \mathrm{Tc}$ pertechnetate. ${ }^{10}{ }^{11}$ Using the dual headed gamma camera, the system performed a complete $360^{\circ}$ orbit around the patient over 16 minutes. Images were acquired into a $128 \times 128$ matrix every $6^{\circ}$ at five seconds per image. After completion of the acquisition, images were summed to improve counting statistics and reconstructed using filtered back projection (Ramp-Butterworth filter, order 10, cutoff 0.45 Nyquist) to produce transaxial images of the stomach.

For estimations of gastric volumes, the transaxial SPECT images were transferred via Interfile to a dedicated Unix workstation. Stomach volume measurements were obtained using the ANALYZE PC 2.5 (Biomedical Imaging Resource, Mayo Foundation, Rochester, Minnesota, USA) software system, ${ }^{17}$ which has been used previously in volumetric imaging studies. ${ }^{11}{ }^{18} 19$ To measure gastric volume, it was necessary to identify the stomach on the transaxial SPECT images and separate it from the background noise. This was accomplished using a semiautomated segmentation algorithm (Object Extractor, ANALYZE PC 2.5) that requires the user to identify an appropriate seed point and greyscale threshold. Three dimensional renderings of the stomach were produced (fig 1). Any extraneous structures, such as the upper duodenum or a kidney, in close proximity to the posterior aspect of the stomach (which had not been removed in the segmentation algorithm) were removed manually.

Radiation exposure was within permissible ranges for research and clinical studies. The effective radiation dose to the body was 619 mrem, which is approximately 2.7 times the radiation exposure from a full gastrointestinal transit measurement by scintigraphy. Organ exposures and effective dose equivalents have been published elsewhere. ${ }^{14}$

\section{SPECT measurements of defined balloon volumes in vitro}

An infinitely compliant polyethylene balloon was filled with known volumes of ${ }^{99 m}$ Tc DTPA labelled water (range 150-800 $\mathrm{ml}$ ) and placed in a water bath. Tomographic images were acquired using the large field of view dual headed gamma camera system (SMV SPECT System) described above. The gamma camera performed a $360^{\circ}$ orbit around the balloon suspended in the water bath, and volume measurements were performed as outlined above. Volume measurements by SPECT were then compared with the known volumes in the balloon.

\section{Simultaneous assessment of stomach function using gastric barostat and SPECT}

Measurements were performed simultaneously using a barostatic balloon within the stomach and the SPECT imaging technique described above (fig 2).

Following an overnight fast, a double lumen barostat assembly with an infinitely compliant polyethylene balloon (maximum volume $1000 \mathrm{ml}$ ) was placed such that the balloon was in the proximal stomach. The balloon was tied onto two metal rings incorporated into the tube assembly. The tube was inserted to $60 \mathrm{~cm}$, and the participant assumed the supine position. The barostat balloon was inflated to $300 \mathrm{ml}$, and the 


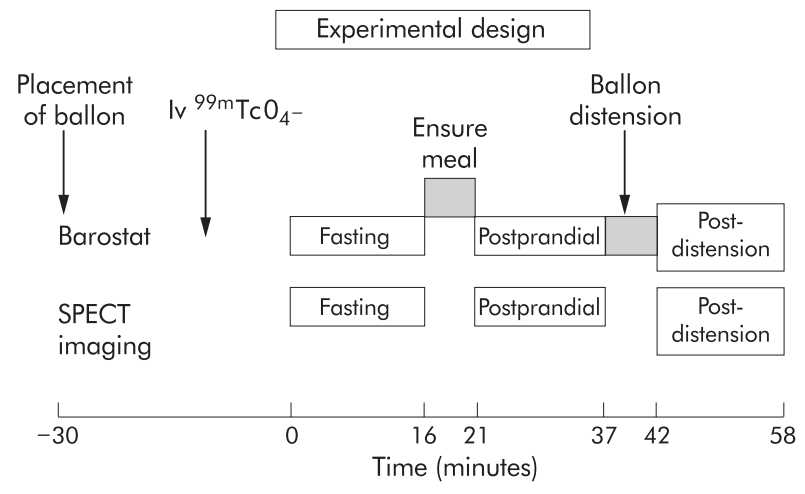

Figure 2 Experimental design for simultaneous assessment of volume changes using the gastric barostat and single photon emission computed tomography (SPECT).

tube was pulled back into the proximal stomach until encountering resistance at the gastro-oesophageal junction. The position of the inflated balloon was checked under fluoroscopy to ensure it was located in the proximal stomach. The tube was anchored by taping it to the chin. The baseline operating pressure (intraballoon pressure $2 \mathrm{~mm} \mathrm{Hg}$ above the pressure at which volumetric variations due to respiratory changes were observed) was identified. The air velocity for inflation or deflation with the rigid piston Mayo barostat was $25 \mathrm{ml} / \mathrm{s}$.

An equilibration period of 30 minutes at the baseline operating pressure was allowed before formal measurements of fasting balloon volumes were recorded. Twenty minutes into the equilibration period, $10 \mathrm{mCi}{ }^{99 m} \mathrm{Tc}$ sodium pertechnetate was administered intravenously allowing 10 minutes for uptake of the isotope by the gastric mucosa prior to assessing fasting gastric volume. During a 16 minute time period, we measured fasting gastric volume using simultaneous SPECT imaging with the intragastric balloon under barostatic conditions. On completion of the fasting SPECT scan, $300 \mathrm{ml}$ Ensure (Ross Products, Division of Abbott Laboratories, Columbus, Ohio, USA; $316 \mathrm{kcal}, 7.6 \mathrm{~g}$ fat, $50.6 \mathrm{~g}$ carbohydrate, and $11.4 \mathrm{~g}$ protein) were administered through the distal end of the intragastric tube over a five minute period.

The postprandial response to the liquid nutrient meal was measured simultaneously by the two techniques. Using the gastric barostat, the accommodation response was the postprandial change in intragastric balloon volume at the preset operating pressure. The mean balloon volume recorded during the first 16 minutes post-meal was used as the postprandial volume. This time period was based on numerous barostat based studies in the literature that have demonstrated that, in healthy subjects, the accommodation (volume) response to a meal typically reaches a peak within 10 minutes after completion of meal ingestion, and is maintained at the maximum level $\pm 5 \%$ for at least 30 minutes. ${ }^{1-7}$

Following assessment of the postprandial volume response, the stomach was distended by increasing the barostat balloon volume to $600-700 \mathrm{ml}$, and the pressure was clamped when the volume was at least $600 \mathrm{ml}$. Five minutes later, the post-distension volume of the whole stomach was measured with SPECT for 16 minutes and the average barostat balloon volumes recorded during the 16 minute post-distension period were compared with the gastric volumes measured using SPECT.

\section{Gastric volumes in healthy adults and patients post-fundoplication}

Fasting and postprandial gastric volumes were measured using a protocol similar to that outlined above but without the intragastric barostat balloon. Ten minutes after intravenous injection of $10 \mathrm{mCi}{ }^{99 \mathrm{~m}} \mathrm{Tc}$ pertechnetate, SPECT imaging was performed during fasting and over 32 minutes (two camera orbits) following ingestion of a $300 \mathrm{ml}$ Ensure drink through a straw. Transaxial images of the stomach were rendered with ANALYZE to reconstruct three dimensional images and to measure gastric volumes during the fasting and postprandial periods. The two postprandial volume estimates were averaged. In all cases, the volumes measured over 0-16 and 17-32 minutes differed by less than $10 \%$. Volume changes and ratios between the fasting and postprandial periods were calculated.

\section{Statistical analysis}

Linear regression analysis and computed $R^{2}$ were used to assess the accuracy of SPECT in measuring balloon volumes in vitro. Linear regression analysis was also used to compare in vivo postprandial changes in intragastric balloon volume obtained with the barostat with changes in total gastric volume detected by SPECT. The Wilcoxon signed rank test was used to compare simultaneous barostat and SPECT estimates. The postprandial volume response is presented as the difference between postprandial and fasting volumes measured by barostat and SPECT, and as the ratio of postprandial to fasting volumes. Descriptive statistics were used to summarise gastric volumes in the cohort of healthy volunteers. The Wilcoxon signed rank test was used to compare absolute (fasting and average postprandial) volumes and volume changes between males and females, and to compare volumes in healthy adults with those in postfundoplication patients. We compared gastric volume responses for three age ranges ( $18-30,31-40$, and $41-60$ years) in healthy subjects using one way analysis of covariance including sex and body mass index as covariates.

\section{RESULTS}

\section{SPECT measurement of defined balloon volumes in} vitro

Figure 3 shows the actual volumes and SPECT volume estimates for the water filled balloons. SPECT derived volume estimates were highly accurate and showed a near perfect linear relationship with actual balloon volumes $\left(R^{2}=0.99\right)$.

\section{Simultaneous SPECT and barostat assessment of the stomach}

Table 1 shows fasting and postprandial intragastric balloon and total gastric volumes measured by the barostat and SPECT techniques, respectively. As expected, total gastric volumes measured by SPECT were larger than intragastric balloon volumes obtained for the barostatically controlled balloon. Mean ratios of postprandial to fasting balloon and total gastric volumes measured by barostat and SPECT, respectively, were

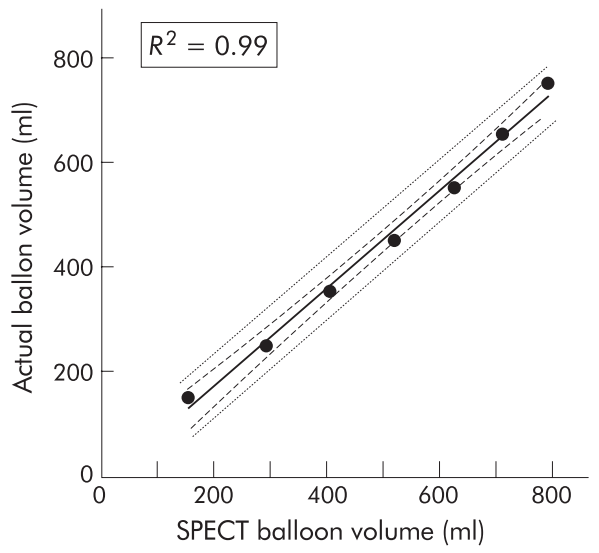

Figure 3 Correlation of known balloon volumes with volumes measured in vitro by single photon emission computed tomography (SPECT). 
Table 1 Gastric volumes by single photon emission computed tomography (SPECT) and barostat effects of meal and distension

\begin{tabular}{|c|c|c|c|}
\hline & Barostat & SPECT & Correlation coefficient \\
\hline \multicolumn{4}{|c|}{ Fasting and postprandial volumes $(m l)(n=16)$} \\
\hline Fasting volume & $238(14)$ & $627(37)$ & $0.60(p=0.02)$ \\
\hline Postprandial volume & $512(25)$ & $1380(37)$ & $0.64(p=0.008)$ \\
\hline Difference & $274(20)$ & $753(47)$ & $0.68(p=0.003)$ \\
\hline Postprandial ratio & $2.2(0.12)$ & $2.3(0.15)$ & $0.8 \quad(p=0.0001)$ \\
\hline \multicolumn{4}{|c|}{ Postprandial and post-distension volumes $(\mathrm{ml})(\mathrm{n}=14)$} \\
\hline Before distention & $515(27)$ & 1387 (39) & \\
\hline After distention & $672(11)$ & $1720(51)$ & \\
\hline Ratio & $1.35(0.07)$ & $1.25(0.04)$ & \\
\hline
\end{tabular}

Table 2 Normative data for gastric volumes measured in 73 healthy volunteers

\begin{tabular}{lccll}
\hline Volume (ml) & $\begin{array}{l}\text { All } \\
(\mathrm{n}=73)\end{array}$ & $\begin{array}{l}\text { Males } \\
(\mathrm{n}=25)\end{array}$ & $\begin{array}{l}\text { Females } \\
(\mathrm{n}=48)\end{array}$ & $\begin{array}{l}\mathrm{p} \text { Value } \\
\text { (males } v \text { females) }\end{array}$ \\
\hline Fasting & $213(6)$ & $215(11)$ & $211(7)$ & 0.5 \\
Postprandial & $698(12)$ & $744(20)$ & $675(14)$ & 0.004 \\
Postprandial minus fasting & $483(12)$ & $521(20)$ & $464(14)$ & 0.01 \\
Postprandial/fasting volume ratio & $3.4(0.1)$ & $3.6(0.2)$ & $3.4(0.1)$ & 0.3 \\
\hline
\end{tabular}

highly comparable $(\mathrm{p}=0.6)$. Figure 4 plots the postprandial ratios, as measured by both techniques $\left(R^{2}=0.7\right)$. Significant positive linear relationships were noted for volume estimates between barostat and SPECT.

Postprandial balloon volumes were 512 (25) $\mathrm{ml}$; the additional balloon distension resulted in a volume increase within the balloon of 157 (78) ml. Balloon distension was associated with an increase in whole stomach volume measured by SPECT. Distension was associated with a mean increase in stomach volume of 333 (49) ml, approximately double the average volume increase measured within the balloon, suggesting the whole stomach responded to the additional distension volume. Post-distention ratios assessed by the barostat and by SPECT were comparable $(p=0.2)$ (table $1)$.

\section{Normative data for healthy adults}

Fasting gastric volumes did not differ between males and females. Postprandial gastric volumes $(p=0.004)$ and postprandial gastric volume changes $(p=0.01)$ were significantly greater in males than females (table 2 ). Age and body mass index did not influence fasting or postprandial gastric volumes. Specifically, gastric volumes and the postprandial response were not significantly different for healthy volunteers aged $18-30$ years $(n=27), 31-40$ years $(n=28)$, and

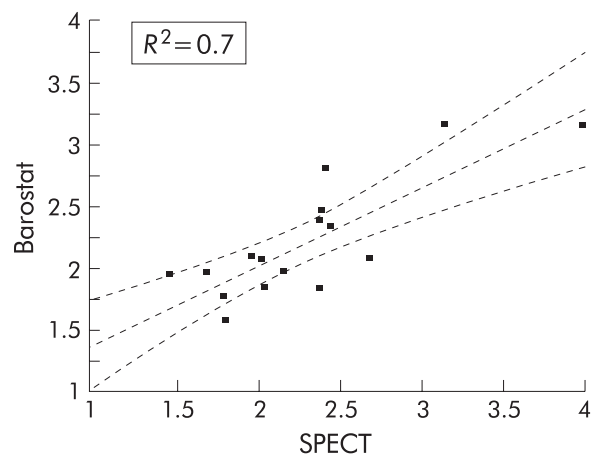

Figure 4 Correlation of postprandial accommodation ratios, as measured by single photon emission computed tomography (SPECT) and gastric barostat.

\begin{tabular}{ll} 
Table 3 Post-fundoplication patients \\
\hline Parameter \\
\hline Fasting volume (ml) & $281(25)^{*}$ \\
Postprandial volume (ml) & $717(37)$ \\
Postprandial minus fasting (ml) & $436(25)$ \\
Postprandial/fasting volume ratio & $2.7(0.2)^{*}$ \\
\hline${ }^{*} p<0.005$ versus controls. &
\end{tabular}

41-60 years $(n=18)$. Body mass index did not significantly impact on the effect of sex on postprandial gastric volumes.

\section{Symptomatic post-fundoplication patients}

Gastric volumes and volume ratios in patients with upper gastrointestinal symptoms post-fundoplication were quite variable (table 3). Fasting gastric volumes were higher in post-fundoplication patients relative to healthy controls $(p<0.005)$. As shown in fig 5 , the postprandial to fasting gastric volume ratios were significantly lower in the postfundoplication group compared with healthy controls $(p=0.003)$. However, it is worth noting that gastric volumes and volume ratios of several of the post-fundoplication patients were well within the normal range.

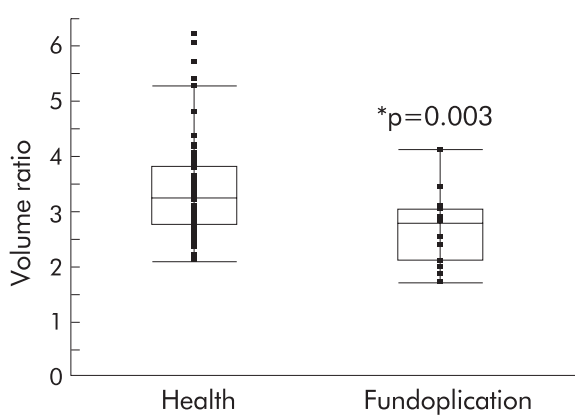

Figure 5 Postprandial to fasting gastric volume ratio in health and post-fundoplication symptomatic patients. Note the overall difference in the two groups but the significant overlap of data for a sizable proportion of patients with data from healthy controls. 


\section{DISCUSSION}

We have shown that the novel SPECT-ANALYZE technique accurately measures known volumes, as demonstrated by the results of the in vitro volume validation protocol. In vivo, SPECT measures total gastric volumes post-meal and postdistension, which are larger than intragastric balloon volumes measured using the barostat method. Postprandial and post-distension ratios (which estimate volume changes) measured by gastric barostat and SPECT techniques were highly comparable.

SPECT does not directly measure gastric tone as it does not evaluate intragastric pressure. Intragastric pressure is homeostatically controlled by changes in gastric tone/tension and equilibration with outside pressure by venting through the belch reflex ${ }^{21}$ which accommodate the hydrostatic pressure from intragastric content and other causes of change in intraabdominal pressure. As the volume and caloric density of the meal were standardised and intra-abdominal pressure is relatively constant within an individual, an increase in gastric volume indirectly reflects a change in gastric tone. In fact, the postprandial to fasting volume ratios measured with SPECT (without knowledge of intragastric pressure) were highly comparable with those measured with the barostatic balloon.

Our previous studies suggested that non-invasive measurement of gastric volume may be a practical surrogate for the invasive assessment of tone with the barostat: SPECT documents as volume changes ${ }^{6}{ }^{16}$ the expected effects of pharmacological agents on gastric tone, as measured by the barostatic balloon with nitrates, ${ }^{22}{ }^{23}$ the motilide erythromycin, ${ }^{24}$ and the $5-\mathrm{HT}_{3}$ antagonist alosetron. ${ }^{15}{ }^{25}{ }^{26}$ Finally, in patients with idiopathic non-ulcer dyspepsia, SPECT showed that approximately $40 \%$ had lower volume increases in response to the standard Ensure meal, ${ }^{13}$ and this proposition coincides with Tack's observation ${ }^{4}$ obtained with the barostatic balloon.

Techniques that do not require intubation to measure gastric volume have no direct effect on gastric relaxation, either by triggering of the swallowing reflex by the tube and swallow induced relaxation or by distension of the stomach with the balloon. A potential advantage of the less invasive SPECT method is the absence of any interference of external imaging with the physiological functions of the stomach. It has been suggested that the gastric barostat balloon may function as a foreign object and could interfere with physiological assessment of gastric function. ${ }^{27}$ Data from our study confirm the observations of Samsom and colleagues ${ }^{27}$ as fasting and postprandial gastric volumes measured by SPECT in healthy subjects in the presence of an intragastric balloon were 2 to 3 fold higher than volumes measured in other healthy subjects in the absence of the balloon. Relaxation within an organ or in anatomically adjacent organs in response to distension at one site is a well established reflex. ${ }^{28}$

The experiments in this study were performed with participants in the supine position because of physical limitations imposed by the SPECT camera. However, fasting and postprandial volumes and ratios measured by the gastric barostat in this study were consistent with values reported from other gastric barostat studies performed in the seated position. ${ }^{1246}$ These data suggest that meal induced changes in intragastric balloon volume measured by barostat were in the physiological range. Simultaneously performed SPECT identified gastric volume changes with a nutrient liquid meal.

A potential limitation of the SPECT technique is radiation exposure which could limit the number of studies in individual patients. Other techniques such as ultrasound, ${ }^{29}$ magnetic resonance imaging, ${ }^{30}$ and volumetric fluid loading $^{41}$ have been proposed as alternative methods to measure gastric accommodation but they have not been completely validated to date.

The present study also characterised normative SPECT data for fasting and postprandial gastric volumes in 73 healthy adults. Females have a significantly lower postprandial volume compared with males, and this difference is not influenced by body mass index in the range tested. The average 10\% sex related difference in postprandial volume is unlikely to be biologically important but it has important implications in planning studies of perturbations for which sex should be included in the randomisation plan. There were no significant differences in gastric volumes for adults up to 60 years of age.

We have also characterised the postprandial change in gastric volume in patients with prior fundoplication and upper gastrointestinal symptoms. As a group, post-fundoplication patients manifested a significant reduction in the postprandial response, confirming results from a study with an intragastric barostatic balloon. ${ }^{7}$ However, several patients had normal volume responses and alternative explanations such as gastric hypersensitivity should be considered in evaluating the cause of symptoms in these patients.

In conclusion, the SPECT-ANALYZE technique accurately measures volume in vitro and can characterise changes in gastric volume in response to a meal and gastric distension. Volume changes (expressed as ratios) with SPECT are comparable with those identified using the gastric barostat. The SPECT technique allows non-invasive visualisation and measurement of the entire stomach volume. This technique should facilitate evaluation of the effects of pharmacological agents on gastric volume and comparisons between health and disease states.

\section{ACKNOWLEDGEMENTS}

This study was supported in part by the General Clinical Research Center grant No RR00585 (Physiology Core) from National Institutes of Health. Dr Bouras received support from the American College of Gastroenterology. Dr Camilleri is supported by grants R01-DK54681 and K24-DK02638 from National Institutes of Health. We thank Ms Cindy Stanislav for secretarial support.

This paper was presented in part at the Annual Meeting of the American Gastroenterological Association (May 2001, Atlanta, GA) and appears in abstract form in Gastroenterology 2001;120:A97, and at the International Symposium for Neurogastroenterology and Motility (November 2001, Madison, WI) and appears in abstract form in Neurogastroenterology and Motility 2001;13:378.

\section{Authors' affiliations}

E P Bouras, S Delgado-Aros, M Camilleri, E J Castillo, D D Burton, G M Thomforde, H J Chial, Clinical Enteric Neuroscience Translational and Epidemiological Research Program, Mayo Clinic and Mayo

Foundation, Rochester, Minnesota, USA

\section{REFERENCES}

1 Azpiroz F, Malagelada JR. Gastric tone measured by an electronic barostat in health and postsurgical gastroparesis. Gastroenterology 1987;92:934-43

2 Salet GA, Samsom M, Roelofs JM, et al. Responses to gastric distension in functional dyspepsia. Gut 1998;42:823-9.

3 Samsom M, Salet GA, Roelofs JM, et al. Compliance of the proximal stomach and dyspeptic symptoms in patients with type I diabetes mellitus. Dig Dis Sci 1995;40:2037-42.

4 Tack J, Piessevaux H, Coulie B, et al. Role of impaired gastric accommodation to a meal in functional dyspepsia. Gastroenterology 1998;115:1346-52

5 Thumshirn M, Camilleri M, Hanson RB, et al. Gastric mechanosensory and lower esophageal sphincter function in rumination syndrome. Am J Physiol 1998;275:G314-21.

6 Thumshirn M, Camilleri M, Saslow SB, et al. Gastric accommodation in non-ulcer dyspepsia and the roles of Helicobacter pylori infection and vagal function. Gut 1999;44:55-64

7 Wijnhoven BP, Salet GA, Roelofs JM, et al. Function of the proximal stomach after Nissen fundoplication. Br J Surg 1998;85:267-71.

8 Berardi C, Twardock AR, Wheaton LG, et al. Nuclear imaging of the stomach of healthy dogs. Am J Vet Res 1991;52:1081-8.

9 O'Connor MK, O'Connell R, Keane FB, et al. The relationship between technetium $99 \mathrm{~m}$ pertechnetate gastric scanning and gastric contents. $\mathrm{Br} J$ Radiol 1983;56:817-22.

10 Prather CM, Foley MK, Geisler KL, et al. A novel, enhanced scintigraphic image analysis of gastric motor physiology using ANALYZE ${ }^{T M}$. Gastroenterology 1996;1 10:A739. 
11 Kuiken SD, Samsom M, Camilleri M, et al. Development of a test to measure gastric accommodation in humans. Am J Physiol 1999;277:G1217-21

12 Delgado-Aros S, Kim D-Y, Burton DD, et al. Effect of GLP-1 on gastric volume, emptying, maximum volume ingested and postprandial symptoms in humans. Am J Physiol Gastrointest Liver Physiol 2002;282:G424-31

13 Kim D-Y, Camilleri M, Murray JA, et al. Is there a role for gastric accommodation and satiety in asymptomatic obese people? Obesity Res 2001;9:655-61.

$14 \mathrm{Kim}$ D-Y, Delgado-Aros S, Camilleri M, et al. Noninvasive measuremen of gastric accommodation in patients with idiopathic nonulcer dyspepsia. Am J Gastroenterol 2001;96:3099-105.

15 Kuo B, Camilleri M, Burton D, et al. Effects of $5-\mathrm{HT}_{3}$ antagonism on postprandial gastric volume and symptoms in humans. Aliment Pharm Ther 2002; 16:225-33

16 Liau S-S, Camilleri M, Kim D-Y, et al. Pharmacological modulation of human gastric volumes demonstrated noninvasively using SPECT imaging. Neurogastroenterol Motil 2001;13:533-42.

17 Hanson DP, Robb RA, Aharon S, et al. New software tool kits fo comprehensive visualization and analysis of three-dimensional multimodal biomedical images. J Digit Imaging 1997; 10:229-30.

18 Jack CR, Peterson RC, Xu Y, et al. Rate of medial temporal lobe atrophy in typical aging and Alzheimer's disease. Neurology 1998;51:993-9.

19 O'Brien TJ, O'Connor MK, Mullan BP, et al. Subtraction ictal SPET co-registered to MRI in partial epilepsy: description and technical validation of the method with phantom and patient studies. Nucl Med Comm 1998;19:31-45.

20 Madsen JL. Effects of gender, age, and body mass index on gastrointestinal transit times. Dig Dis Sci 1992;37:1548-53.
21 Wyman JB, Dent J, Heddle R, et al. Control of belching by the lower oesophageal sphincter. Gut 1990;31:639-46

22 Schuurkes JA, Meulemans AL. Nitric oxide and gastric relaxation. Dig Dis Sci 1994;39(12 suppl):79-81S

23 Thumshirn M, Camilleri M, Choi MG, et al. Modulation of gastric sensory and motor functions by nitrergic and alpha2-adrenergic agents in humans. Gastroenterology 1999;1 16:573-85.

24 Bruley des Varannes S, Parys V, Ropert A, et al. Erythromycin enhances fasting and postprandial proximal gastric tone in humans. Gastroenterology 1995;109:32-9.

25 Wilmer A, Coulie B, Tack J, et al. Effect of the 5- $\mathrm{HT}_{3}$ receptor antagonist ondansetron, on gastric accommodation and on perception of gastric distension after a meal. Gastroenterology 1996;1 10:A781.

26 Zerbib F, Bruley des Varannes S, Oriola RC, et al. Alosetron does not affect the visceral perception of gastric distension in healthy subjects. Aliment Pharm Ther 1994;8:403-7.

27 Samsom M. Hauskens T, Mundt M. Gastric accommodation is influenced by the presence of an intragastric balloon. Gastroenterology 2000;118:A621.

28 Kreulen DL, Muir TC, Szurszewski JH. Peripheral sympathetic pathways to gastroduodenal region of the guinea pig. Am J Physiol 1983;245:G369-75.

29 Tefera S, Gilja OH, Hatlebakk JG, et al. Gastric accommodation studied by ultrasonography in patients with reflux esophagitis. Dig Dis Sci 2001;46:618-25

30 Feinle $C$, Kunz $P$, Boesiger $P$, et al. Scintigraphic validation of a magnetic resonance imaging method to study gastric emptying of a solid meal in humans. Gut 1999:44:106-11.

31 Tosetti C, Salvioli B, Stanghellini V, et al. Reproducibility of a water load test in healthy subjects and symptom profile compared to patients with functional dyspepsia. Gastroenterology 1999;116:A336.

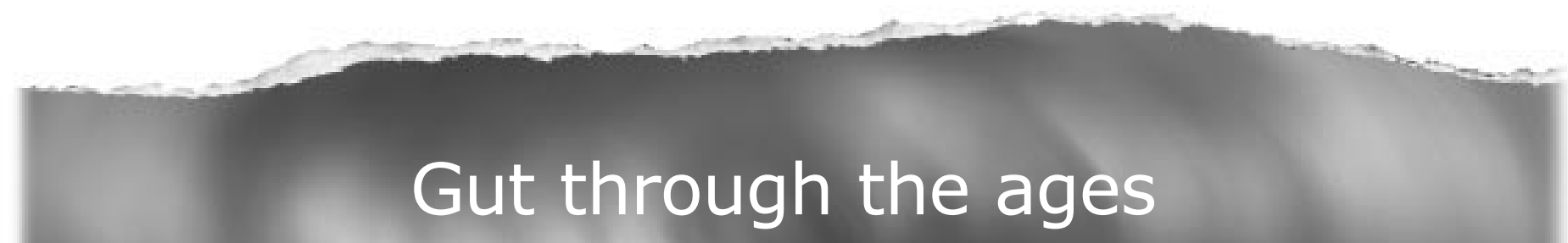

Browse the Archive

Gut online has an archive of content dating back to 1966.

Full text from 1997; abstracts from 1975; table of contents from 1966

www.gutjnl.com 